This document is the accepted manuscript version of the following article: Quan, Y., Kohlbrecher, J., Hautle, P., \& Michels, A. (2020). Defect-induced DzyaloshinskiiMoriya interaction in a nanocrystalline two-phase alloy. Journal of Physics:

Condensed Matter, 32(28), 285804 (8 pp.). https://doi.org/10.1088/1361-648X/ab80f8

\title{
Defect-Induced Dzyaloshinskii-Moriya Interaction in a Nanocrystalline Two-Phase Alloy
}

\author{
Yifan Quan ${ }^{1}$, Joachim Kohlbrecher ${ }^{1}$, Patrick Hautle ${ }^{1}$ and \\ Andreas Michels ${ }^{2}$ \\ ${ }^{1}$ Paul Scherrer Institute, 5232 Villigen PSI, Switzerland \\ 2 Department of Physics and Materials Science, University of Luxembourg, 162A \\ Avenue de la Faïencerie, L-1511 Luxembourg, Grand Duchy of Luxembourg \\ E-mail: yifan.quan@psi.ch
}

\begin{abstract}
The Dzyaloshinskii-Moriya interaction (DMI) is believed to be operative in low-symmetry crystal structures lacking space-inversion symmetry. However, already in 1963, Arrott pointed out that even in a high-symmetry lattice, where the DMI would normally vanish, this interaction is present in the vicinity of any lattice defect. Based on these considerations and recent theoretical work, first experimental studies of the impact of the DMI on the spin-polarized magnetic small-angle neutron scattering (SANS) of polycrystalline magnets exhibiting a large density of microstructural defects have been performed. They demonstrated that an asymmetry in the difference between the two polarized SANS cross sections is induced by the DMI in nanocrystalline terbium and holmium as well as in mechanicallydeformed microcrystalline cobalt. Here, we present a more complicated case, the nanocrystalline magnetically-textured soft magnet Vitroperm $\left(\mathrm{Fe}_{73} \mathrm{Si}_{16} \mathrm{~B}_{7} \mathrm{Nb}_{3} \mathrm{Cu}_{1}\right)$, where the interface between the FeSi nanoparticles and the amorphous magnetic matrix serves as the defect. The SANS cross section exhibits the polarization-dependent asymmetric term originating from the DMI. The effect has a magnetic field dependence and is less pronounced at higher fields until it eventually vanishes at full saturation. The result supports the generic relevance of the DMI for the magnetic structure of defect-rich ferromagnets. Furthermore, it shows that polarized SANS is a particularly powerful tool for investigating defect-induced DMI, which is a consequence of the unique dependence of the SANS cross section on the chiral interactions.
\end{abstract}




\section{Introduction}

The Dzyaloshinskii-Moriya interaction (DMI) [1, 2] is an important ingredient for the stabilization of various types of skyrmion textures (see, e.g., [3, 4, 5, 6, 7, 8, 9] and references therein). The origin of the DMI is due to the relativistic spin-orbit coupling and in low-symmetry crystal structures lacking inversion symmetry it gives rise to antisymmetric magnetic interactions. In most of the studies published so far the origin of the DMI is related to the noncentrosymmetric crystal structures of the materials under study, or to the breaking of structural inversion symmetry at the interfaces in ultrathin film architectures.

A less studied aspect of the DMI is related to a prediction by Anthony Arrott from 1963 [10]. He pointed out that the DMI is present in the vicinity of any lattice defect and that it gives rise to inhomogeneous magnetization states: For two magnetic ions which are ferromagnetically coupled by the isotropic exchange interaction, the DMI, when acting on the exchange path, produces an antiferromagnetic component, while for the two ions being antiferromagnetically aligned by isotropic exchange, the DMI causes a ferromagnetic component. In a sense, microstructural defects act as a source of additional local chiral interactions, similar to the above mentioned intrinsic DMI in noncentrosymmetric crystal structures. Based on the long-ranged Ruderman-KittelKasuya-Yosida Hamiltonian amended by an additional DMI term that describes the spin-orbit scattering of the conduction electrons by nonmagnetic impurities, Fert and Levy $[11,12,13]$ have shown that the effect of the DMI can explain the enhancement of the anisotropy fields observed in certain spin glasses and that the magnitude of the DMI term can be quite large, about $10-20 \%$ of the unperturbed ground state energy. Therefore, it is important to realize that defect-induced DMI is generally operative in polycrystalline and disordered materials, even in high-symmetry lattices, where the 'usual' intrinsic DMI term vanishes. This point of view has also been adopted by Fedorov et al. [14], who studied the impact of torsional-strain-induced DMI couplings near dislocations on the helix domain populations in Ho metal. Similarly, Lott et al. [15] investigated the field-induced chirality in the helix structure of Dy/Y multilayer films and provided evidence for interface-induced DMI. Beck and Fähnle [16] combined abinitio density functional electron theory with a micromagnetic model to study the DMI vectors arising from a fabrication-induced perpendicular strain gradient in a film of bcc Fe. Kitchaev et al. [17] theoretically showed that a DMI may arise in a material with any symmetry when coupled to a strain field. Kim et al. [18] have investigated GdFe-Co amorphous ferrimagnetic alloys and report the observation of a bulk DMI. An asymmetric distribution of elemental content (composition gradient) is supposed to be at the origin of the broken inversion symmetry in the ferrimagnetic layer. Butenko and Rößler [19] have developed a continuum micromagnetic model for dislocationinduced DMI couplings. These authors considered a disk-like film element with a screw dislocation at its center and showed that the associated defect-induced DMI leads to a chirality selection of the vortex state. 
With the above described scenario in mind, the impact of the DMI on the spin-polarized small-angle neutron scattering (SANS) cross section of defect-rich polycrystalline materials - nanocrystalline $\mathrm{Tb}$ and Ho as well as mechanically-deformed Co-has recently been investigated [20, 21]. Polarized SANS is one of the very few techniques that are suitable for studying defect-related DMI, since the scattering cross section contains a purely magnetic term which depends exclusively on the chiral interactions $[14,22]$. The signature of the DMI is an asymmetry in the difference between the two SANS cross sections measured for neutrons polarized parallel and antiparallel to the magnetic guide-field direction [21].

In this paper we present the results of a polarized neutron study of the commercial soft magnetic material Vitroperm $\left(\mathrm{Fe}_{73} \mathrm{Si}_{16} \mathrm{~B}_{7} \mathrm{Nb}_{3} \mathrm{Cu}_{1}\right)$. This alloy, which consists of FeSi nanoparticles (with an ordered $\mathrm{DO}_{3}$ superlattice structure [23]) that are dispersed in an amorphous magnetic matrix, was annealed in the presence of an applied magnetic field and, therefore, possesses a macroscopic texture axis [24, 25]. Compared to the previously studied systems ( $\mathrm{Tb}$, Ho, Co [21]), the Vitroperm sample exhibits the additional complexity that the magnetic microstructure is decorated by jumps in the magnetization magnitude at internal particle-matrix boundaries; in other words, the saturation magnetization $M_{s}$ varies as a function of the position $\mathbf{r}$ inside the material. This gives rise to dipole-field-induced spin disorder and a concomitant clover-leaf-shaped angular anisotropy in the magnetic SANS cross section (e.g., [26, 27, 28]). The analysis of the magnetic-field-dependent scattering asymmetry allows one to estimate the DMI strength, which is presumably related to the broken symmetry at the particle-matrix interfaces in the nanocrystalline material.

\section{Experimental}

\subsection{Sample}

Commercial grade Vitroperm $\left(\mathrm{Fe}_{73} \mathrm{Si}_{16} \mathrm{~B}_{7} \mathrm{Nb}_{3} \mathrm{Cu}_{1}\right)$ with a nominal permeability of $\mu=$ $1.5 \times 10^{5}$ and a saturation magnetization of $\mu_{0} M_{s}=1.2 \mathrm{~T}[24,25]$ was supplied by Vacuumschmelze, Hanau, Germany. The sample was investigated in the as-received state. The microstructure consists of a distribution of FeSi nanograins in an amorphous magnetic matrix. The alloy has a macroscopic texture, which was induced by magneticfield-annealing at about $500{ }^{\circ} \mathrm{C}$ (performed by Vacuumschmelze). From the Guinier analysis of the scattering data (see below) we have estimated a mean particle radius of $R=(6 \pm 1) \mathrm{nm}$. The room-temperature magnetization curve of the Vitroperm sample is depicted in Fig. 1 and shows that saturation is reached for applied fields larger than $\sim 10 \mathrm{mT}$ (essentially the demagnetization field of the sample). This type of material has previously been investigated by SANS [29, 30], where the focus was set on the orientation of the net magnetization of the domains and on the length scale characterizing the internal spin disorder within the domains. The SANS sample consists of a stack of 20 sheets (individual sheet thickness: $30 \mu \mathrm{m}$ ) with an area of $25 \times 35 \mathrm{~mm}^{2}$. 


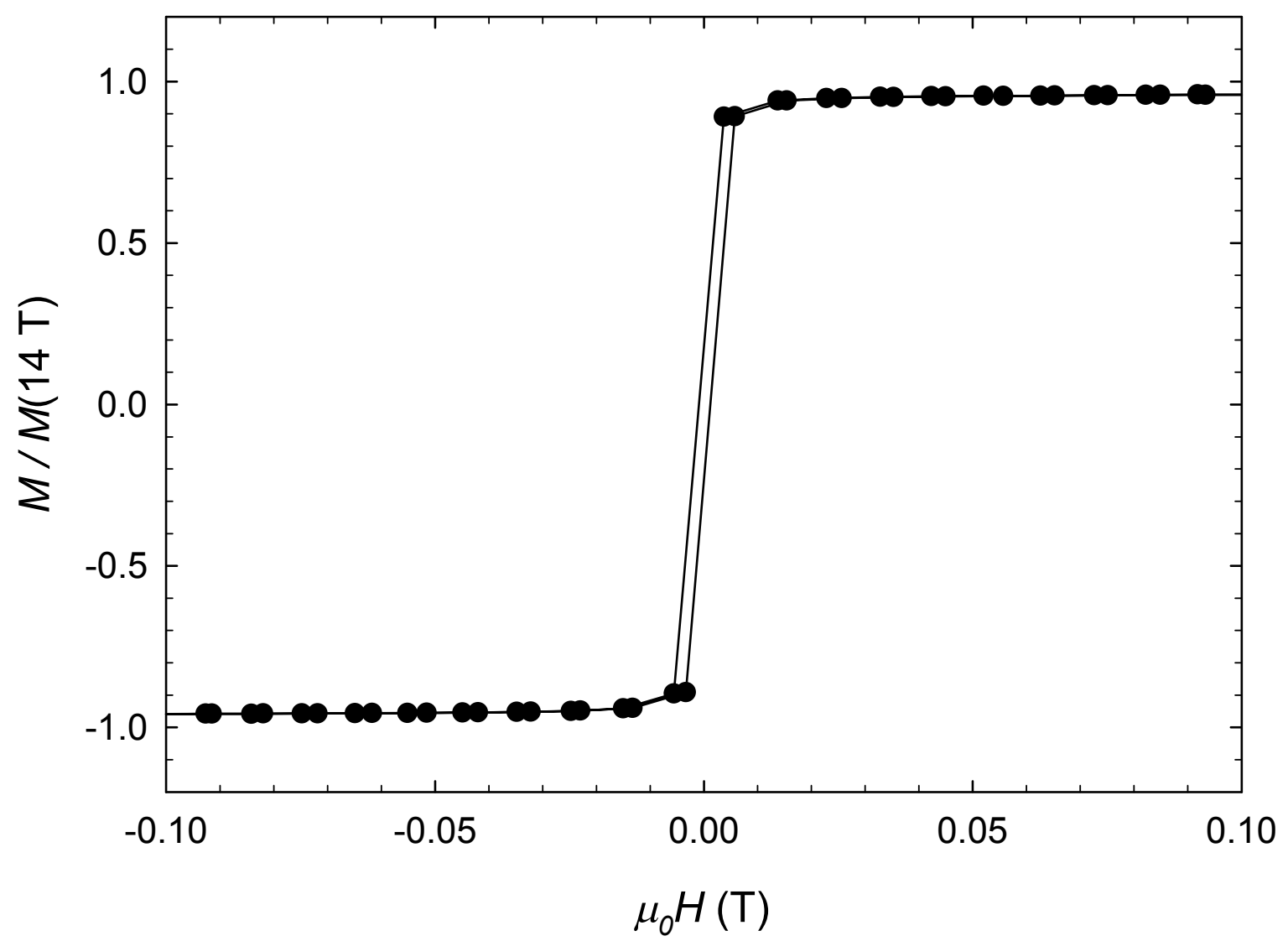

Figure 1: Normalized room temperature magnetization curve of Vitroperm $\left(\mathrm{Fe}_{73} \mathrm{Si}_{16} \mathrm{~B}_{7} \mathrm{Nb}_{3} \mathrm{Cu}_{1}\right)$.

The total sample thickness has been optimized by performing a depolarization analysis, the results of which will be published elsewhere.

\section{2. $S A N S$}

The SANS experiments were performed at the SANS I instrument [31] at the continuous spallation neutron source SINQ at the Paul Scherrer Institute (PSI), Switzerland. The incident neutron beam had a mean wavelength of $\lambda=6 \AA$ with a wavelength spread of $\Delta \lambda / \lambda=10 \%$ (FWHM) and was polarized by means of a $\mathrm{V}$-shaped Fe/Si supermirror transmission polarizer to $P=0.98$. The neutron polarization could be reversed by means of an adiabatic spin flipper with an efficiency of $\epsilon=99 \%$. The sample was kept at room temperature and mounted in an electromagnet. The external magnetic field $\mathbf{H}_{0}$ was applied perpendicular to the wave vector $\mathbf{k}_{0}$ of the incident neutron beam and parallel to the sample's easy axis. The depolarization of the transmitted beam by the sample was checked with a spin analyzer based on polarized protons [32]. For a stack of 20 sheets of the sample at $20 \mathrm{mT}$, the polarization of the transmitted neutron beam was 
reduced to $75 \%$. The scattering intensities for spin-up and spin-down incident neutrons $I^{+}$and $I^{-}$were then measured at a detector distance of $11 \mathrm{~m}$. SANS experiments with a polarized incident beam only, and no spin analysis of the scattered neutrons, are commonly denoted as "half-polarized" SANS. Scattering patterns were recorded for different magnetic-field values, where for each measurement the sample was first saturated by a field of $0.9 \mathrm{~T}$ in order to follow the same magnetic hysteresis curve. As shown below, the difference between the two intensities gives access to the defect-induced DMI.

\section{Polarized SANS cross section}

Neglecting nuclear spin-incoherent SANS, the two half-polarized elastic differential SANS cross sections for the scattering geometry where the incident neutron beam is perpendicular to the externally applied magnetic field $\left(\mathbf{k}_{0} \perp \mathbf{H}_{0}\right)$ can be expressed as $[9,33,34]$ :

$$
\begin{aligned}
\frac{d \Sigma^{ \pm}}{d \Omega}= & \frac{8 \pi^{3}}{V} b_{H}^{2}\left[b_{H}^{-2}|\widetilde{N}|^{2}+\left|\widetilde{M}_{x}\right|^{2}+\left|\widetilde{M}_{y}\right|^{2} \cos ^{2} \theta+\left|\widetilde{M}_{z}\right|^{2} \sin ^{2} \theta\right. \\
& -\left(\widetilde{M_{y}} \widetilde{M}_{z}^{*}+\widetilde{M}_{y}^{*} \widetilde{M}_{z}\right) \sin \theta \cos \theta+P\left(2 \epsilon^{ \pm}-1\right) b_{H}^{-1}\left(\widetilde{N} \widetilde{M}_{z}^{*}+\widetilde{N}^{*} \widetilde{M}_{z}\right) \sin ^{2} \theta \\
& \left.-P\left(2 \epsilon^{ \pm}-1\right) b_{H}^{-1}\left(\widetilde{N} \widetilde{M}_{y}^{*}+\widetilde{N}^{*} \widetilde{M}_{y}\right) \sin \theta \cos \theta+i P\left(2 \epsilon^{ \pm}-1\right) \chi\right]
\end{aligned}
$$

where the chiral function $\chi(\mathbf{q})$ is given by

$$
\begin{aligned}
\chi(\mathbf{q})= & \left(\widetilde{M}_{x} \widetilde{M}_{y}^{*}-\widetilde{M}_{x}^{*} \widetilde{M}_{y}\right) \cos ^{2} \theta \\
& -\left(\widetilde{M}_{x} \widetilde{M}_{z}^{*}-\widetilde{M}_{x}^{*} \widetilde{M}_{z}\right) \sin \theta \cos \theta .
\end{aligned}
$$

In Eq. (1), $V$ is the scattering volume, the constant $b_{H}=2.91 \times 10^{8} \mathrm{~A}^{-1} \mathrm{~m}^{-1}$ relates the atomic magnetic moment $\mu_{a}$ to the atomic magnetic scattering length $b_{m}=b_{H} \mu_{a}[35,9]$, $\widetilde{N}(\mathbf{q})$ and $\widetilde{\mathbf{M}}(\mathbf{q})=\left\{\widetilde{M}_{x}, \widetilde{M}_{y}, \widetilde{M}_{z}\right\}$ denote, respectively, the Fourier transforms of the nuclear scattering length density and of the magnetization vector field $\mathbf{M}(\mathbf{r})=$ $\left\{M_{x}, M_{y}, M_{z}\right\}, \theta$ is the angle between $\mathbf{H}_{0}=H_{0} \mathbf{e}_{z}$ and $\mathbf{q}$, so that $\mathbf{q} \sim q\{0, \sin \theta, \cos \theta\}$ in the small-angle approximation; $\epsilon^{+}=0$ for flipper off and $\epsilon^{-}=\epsilon$ for flipper on. Then the difference $\Delta \Sigma$ between flipper-on $(-)$ and flipper-off $(+)$ SANS cross sections evaluates to:

$$
\begin{aligned}
\Delta \Sigma & =\frac{d \Sigma^{-}}{d \Omega}-\frac{d \Sigma^{+}}{d \Omega} \\
& =\frac{8 \pi^{3}}{V} b_{H}^{2} P \epsilon \times\left[2 b_{H}^{-1}\left(\widetilde{N} \widetilde{M}_{z}^{*}+\widetilde{N}^{*} \widetilde{M}_{z}\right) \sin ^{2} \theta+2 i \chi\right] .
\end{aligned}
$$

\section{Micromagnetic background}

The difference $\Delta \Sigma$ between spin-up and spin-down SANS cross sections contains (for $\left.\mathbf{k}_{0} \perp \mathbf{H}_{0}\right)$ the contribution due to the chiral function $2 i \chi(\mathbf{q})$ [second term in Eq. (4)]. 
As was shown in [20] using micromagnetic continuum theory, the following expression for $2 i \chi(\mathbf{q})$ is obtained:

$$
2 i \chi(\mathbf{q})=-\frac{2 \widetilde{H}_{p}^{2} p^{3}\left(2+p \sin ^{2} \theta\right) l_{D} q \cos ^{3} \theta+4 \widetilde{M}_{z}^{2} p(1+p)^{2} l_{D} q \sin ^{2} \theta \cos \theta}{\left(1+p \sin ^{2} \theta-p^{2} l_{D}^{2} q^{2} \cos ^{2} \theta\right)^{2}}
$$

where $\widetilde{H}_{p}^{2}\left(q \xi_{H}\right)$ denotes the magnitude square of the anisotropy-field Fourier coefficient, and $\widetilde{M}_{z}^{2}\left(q \xi_{M}\right)$ is the Fourier coefficient of the longitudinal magnetization. These functions characterize the strength and spatial structure of, respectively, the magnetic anisotropy field $\mathbf{H}_{p}(\mathbf{r})$ (with correlation length $\xi_{H}$ ) and of the local saturation magnetization $M_{s}(\mathbf{r})$ (with correlation length $\left.\xi_{M}\right)$. The function $p\left(q, H_{i}\right)=M_{s} /\left[H_{i}(1+\right.$ $\left.\left.l_{H}^{2} q^{2}\right)\right]$ is a known function of $q$ and of the internal field $H_{i}=H_{0}-N M_{s}$ $\left(N\right.$ : demagnetizing factor), while $l_{H}\left(H_{i}\right)=\sqrt{2 A /\left(\mu_{0} M_{s} H_{i}\right)}$ and $l_{D}=2 D /\left(\mu_{0} M_{s}^{2}\right)$ denote the micromagnetic length scales which characterize, respectively, the size of the inhomogeneously magnetized regions around defects and the range of the DMI (A: exchange-stiffness constant; $D$ : DMI constant). A graphical representation of the asymmetric function $-2 i \chi(\mathbf{q})$ is given in Fig. 6 in [20]. At small fields, when Eq. (5) is dominated by the anisotropy term $\propto \widetilde{H}_{p}^{2}$, the function $-2 i \chi(\mathbf{q})$ is enhanced parallel to the field axis $\left(\theta=0^{\circ}\right.$ and $\left.180^{\circ}\right)$, while at larger fields, when the magnetostatic term $\propto \widetilde{M}_{z}^{2}$ plays a more important role, $-2 i \chi(\mathbf{q})$ exhibits extrema roughly along the detector diagonals.

We note that $\chi(\mathbf{q})$ vanishes at complete magnetic saturation $\left(M_{x}=M_{y}=0\right)$, or for purely real-valued magnetization Fourier components (irrespective of the value of the field). For the case that the magnetization distribution is an even function of the position, i.e., $\mathbf{M}(\mathbf{r})=\mathbf{M}(-\mathbf{r})$, the corresponding Fourier transform $\widetilde{\mathbf{M}}(\mathbf{q})$ is also an even and real-valued function, with the consequence that $\chi(\mathbf{q})$ vanishes. On the other hand, if $\mathbf{M}(\mathbf{r})$ is an odd function, then $\widetilde{\mathbf{M}}(\mathbf{q})$ is an odd and imaginary function [36], and $\chi(\mathbf{q})$ is nonzero.

Along the horizontal direction $\left(\theta=0^{\circ}\right.$ and $\left.180^{\circ}\right)$, the nuclear-magnetic interference term $\propto \widetilde{N} \widetilde{M}_{z} \sin ^{2} \theta$ vanishes and Eq. (5) becomes:

$$
2 i \chi\left(q, H_{i}\right)=\mp \frac{4 \widetilde{H}_{p}^{2} p^{3} l_{D} q}{\left(1-p^{2} l_{D}^{2} q^{2}\right)^{2}}
$$

which can be used to analyze experimental data. In our analysis the anisotropy-field Fourier coefficient is described by a squared Lorentzian, $\widetilde{H}_{p}^{2}=\left\langle H_{p}^{2}\right\rangle /\left(1+\xi_{H}^{2} q^{2}\right)^{2}$, where $\left\langle H_{p}^{2}\right\rangle$ is the mean-square anisotropy field, and $\xi_{H}$ denotes the correlation length of the anisotropy field. For an ideal nanocrystalline ferromagnet, the anisotropy field jumps randomly at the interface due to the change of the magnetization. The correlation length $\xi_{H}$ is expected to be related to the average particle size.

\section{Experimental results}

Information on the particle size can be extracted from the analysis of the total unpolarized SANS cross section $\left(\Sigma_{\text {tot }} \propto \Sigma^{+}+\Sigma^{-}\right)$at magnetic saturation $(0.9 \mathrm{~T})$, 


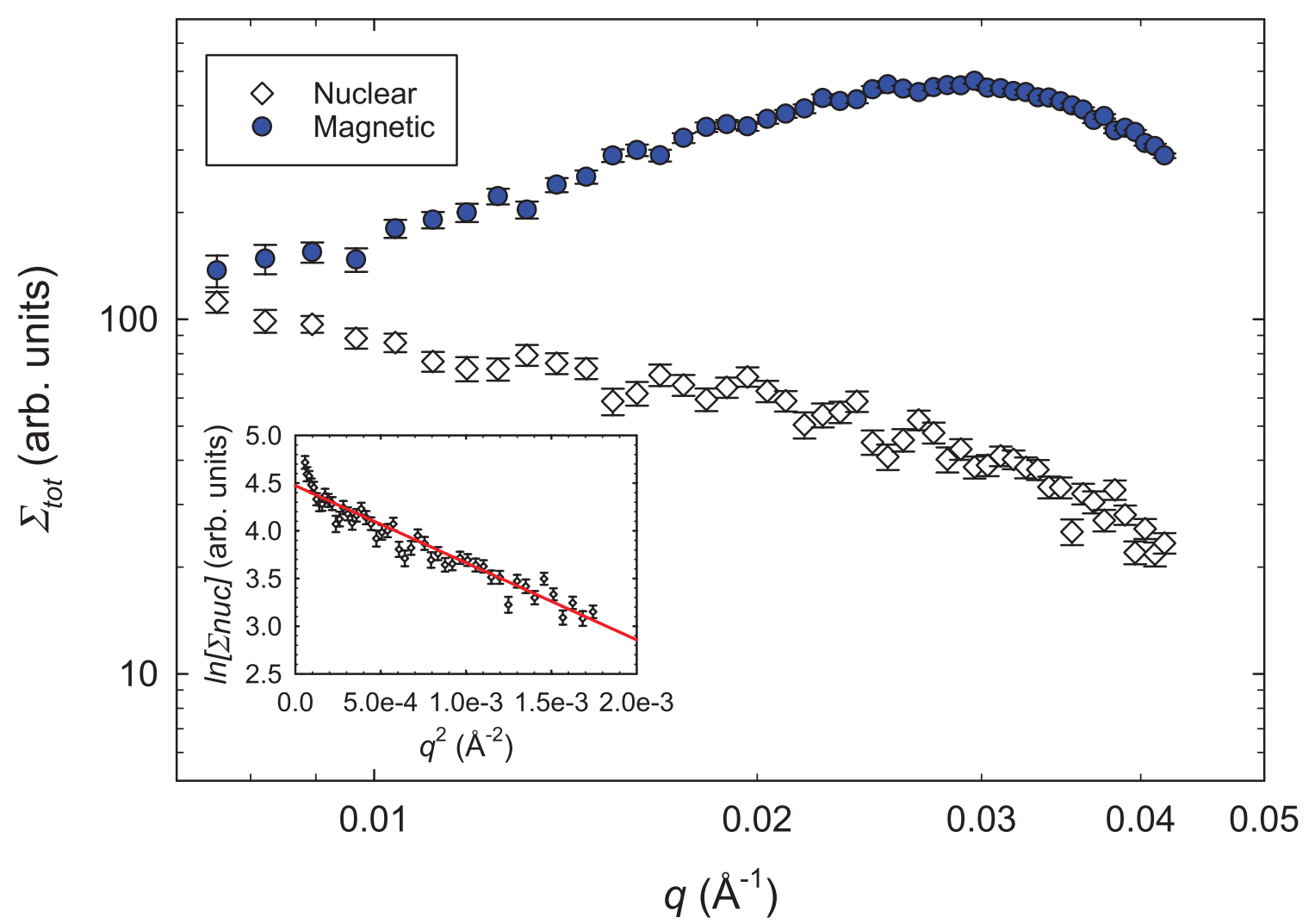

Figure 2: Nuclear and magnetic SANS cross sections at magnetic saturation (log-log scale). A Guinier analysis of the nuclear SANS data (see inset) yields $R_{G}=5 \pm 1 \mathrm{~nm}$.

where the transversal magnetization components vanish and Eq. (1) reduces to $\Sigma_{\text {tot }} \propto$ $|\widetilde{N}(q)|^{2}+\left|\widetilde{M}_{z}(q)\right|^{2} \sin ^{2} \theta$. This expression is fitted to the two-dimensional SANS data, which allows us to separate the nuclear from the dominating magnetic scattering (see Fig. 2). A Guinier analysis is performed on the nuclear SANS and yields an average radius of gyration of the FeSi particles of $R_{G}=(5 \pm 1) \mathrm{nm}$. Assuming a spherical particle shape, the relation $R_{G}^{2}=\frac{3}{5} R^{2}$ suggests an average particle radius of $R=(6 \pm 1) \mathrm{nm}$. This is in agreement with the results of an analysis of wide-angle X-ray diffraction data on a similar sample [29].

The difference between flipper-on and flipper-off SANS data as a function of the applied magnetic field, $\Delta \Sigma$, is shown in Fig. 3. According to the hysteresis loop (Fig. 1) as well as the sufficiently small demagnetizing field of the SANS sample of $H_{d}=N M_{s} \cong 3.5 \mathrm{mT}$, estimated from the sample dimensions [37], all the field values fall into the approach-to-saturation regime, which is a necessary prerequisite for the theory to apply. A strong left-right asymmetry is observed between $14.5 \mathrm{mT}$ and $25 \mathrm{mT}$ and can be related to the predicted expression for the chiral function [Eq. (5)]. This asymmetry is most pronounced along the horizontal direction $\left(\theta=0^{\circ}\right.$ and $\left.180^{\circ}\right)$, 

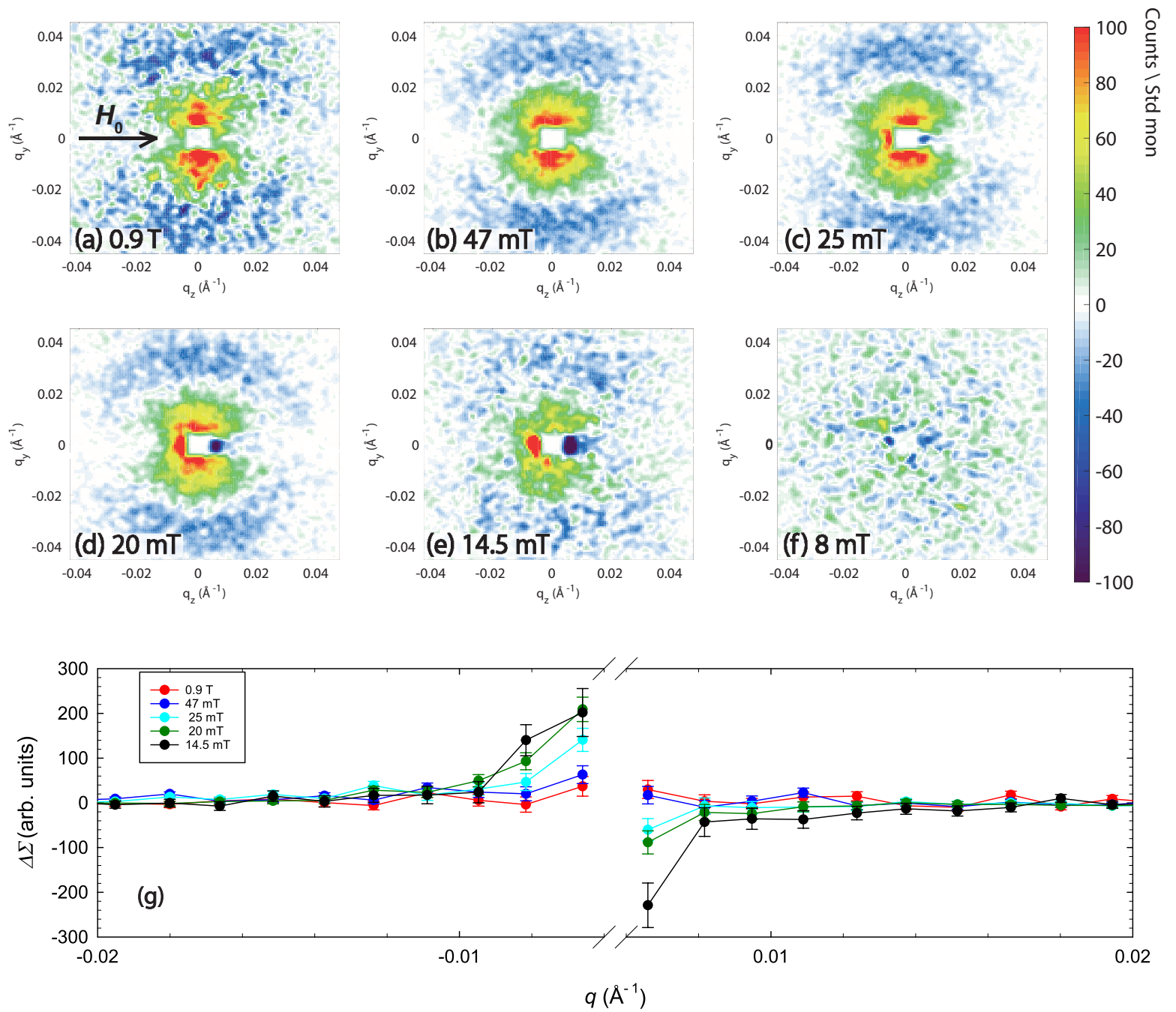

Figure 3: Magnetic field dependence of the DMI asymmetry in Vitroperm $\left(\mathrm{Fe}_{73} \mathrm{Si}_{16} \mathrm{~B}_{7} \mathrm{Nb}_{3} \mathrm{Cu}_{1}\right)$. The external magnetic field $\mathbf{H}_{0} \| \mathbf{e}_{z}$ is applied parallel to the macroscopic easy axis and perpendicular to the incoming neutron beam $\mathbf{k}_{0} \| \mathbf{e}_{x}$. (a)-(f) Two-dimensional flipper-off minus flipper-on data $\Delta \Sigma(\mathbf{q})$ [Eq. (4)]. (g) Azimuthally averaged $\Delta \Sigma(q)\left( \pm 10^{\circ}\right.$ horizontal sector averages) at selected applied magnetic fields (see inset).

which is well explained by the anisotropy-field contribution $\propto-\widetilde{H}_{p}^{2} \cos ^{3} \theta$ in Eq. (5). Additionally, with increasing field, the typical $\sin ^{2} \theta$ anisotropy due to the nuclearmagnetic interference term in Eq. (4) (with maxima along $\theta=90^{\circ}$ and $270^{\circ}$ ) is getting more and more pronounced. Interestingly, the $\widetilde{N} \widetilde{M}_{z} \sin ^{2} \theta$ scattering contribution has a different sign in the high and low- $q$ ranges. This feature can be explained by the different $q$-dependencies of the nuclear and magnetic form factors in our sample (compare Fig. 2). A possible origin for such a difference might be related to the diffusion profile of $\mathrm{Nb}$ around the nanoparticles. On annealing, $\mathrm{Nb}$ is enriched at the interface between particles and matrix and has a strong effect on the magnetic form factor without contributing 
much to the nuclear scattering. Similar features and detailed discussions on the role of $\mathrm{Nb}$ for the SANS of such two-phase alloys can be found in $[38,39,40]$.

Figure $3(\mathrm{~g})$ further displays the angular averages of $\Delta \Sigma$ along the horizontal direction $\left( \pm 10^{\circ}\right)$ as a function of $q$ for different applied magnetic fields. We observe that the DMI asymmetry gets weaker with increasing field until it completely vanishes at full magnetic saturation $(0.9 \mathrm{~T})$. Furthermore, at the lowest field of $8 \mathrm{mT}$, the $\cos ^{3} \theta$ asymmetry together with the $\sin ^{2} \theta$ disappear, which can be explained by the largely misaligned magnetic moments and the formation of a macroscopic domain structure $[29,30]$. In this situation there exists no average magnetization (no axial vector is contained in the system) and correspondingly the SANS cross section is independent of the initial polarization.

To better investigate the asymmetry at $20 \mathrm{mT}$ and $14.5 \mathrm{mT}$, where the effect is strongest, more data has been accumulated with a reduced beam-defining aperture of $9 \times 10 \mathrm{~mm}^{2}$ instead of a $14 \times 10 \mathrm{~mm}^{2}$ aperture. Each spin channel at each field has been measured for 8 hours (typically accumulating more than 6 million total detector counts). The half-polarized SANS cross sections $\Sigma^{+}$and $\Sigma^{-}$in Fig. 4(a)-(d) exhibit a strong signal at very small momentum transfers $\left(q<0.01 \AA^{-1}\right)$ with an elongation along the direction of the applied field. This angular anisotropy is clearly related to misaligned spins in the plane perpendicular to $\mathbf{H}_{0}$ [cf. the term $\left|M_{y}\right|^{2} \cos ^{2} \theta$ in Eq. (1)].

The $\left( \pm 10^{\circ}\right)$ angular averages of $\Delta \Sigma$ along the horizontal direction are very pronounced (see Fig. 5) and can be used to estimate the strength $D$ of the DMI. To do so, the angular averages of $\Delta \Sigma(q)$ of both horizontal $\pm 10^{\circ}$ sectors are combined (taking into account their different signs) and described by Eq. (6) (solid line in Fig. 6). The parameters $\xi_{H}=12 \mathrm{~nm}$ (taken from the Guinier analysis), $\mu_{0} M_{s}=1.2 \mathrm{~T}$ [25], $\mu_{0} H_{i}=\mu_{0}\left(H_{0}-H_{d}\right)=16.5 \mathrm{mT}$ and $11.0 \mathrm{mT}$ (the demagnetizing field $H_{d}$ of the SANS sample is estimated from the sample dimensions and the magnetization [37]), and $A=1.0 \times 10^{-11} \mathrm{~J} / \mathrm{m}[41]$ were held fixed in order to better resolve the DMI constant $D$. Under this constraint we are able to give an upper limit of $D<0.5 \mathrm{~mJ} / \mathrm{m}^{2}$, which is compatible with the results in. [21, 42]. It is difficult to give a better estimate for $D$, since in the studied $q$-range Eq. (6) is less sensitive to $D$ than to the other parameters.

The DMI energy density, $D \mathbf{m} \cdot(\nabla \times \mathbf{m})$ in [20] with $\mathbf{m}(\mathbf{r})$ being the unit magnetization vector, represents a so-called pseudoscalar contribution to the total magnetic energy of a ferromagnet. Pseudoscalar terms change their sign under the inversion operation, and render one type of chirality (corresponding to a negative value of the pseudoscalar) preferred with respect to the other type. Chirality selection happens entirely due to the pseudoscalar terms in the magnetic potential energy. The inversion transformation maps the right-handed structures into the left-handed structures. Therefore, when the inversion symmetry is unbroken, the right and lefthanded structures are equivalent and the DMI vanishes. Moreover, if the defects in the material would be randomly distributed with some defects characterized by a positive $D$ and some others by a negative $D[15]$, then one may expect that the net DMI-induced asymmetry would disappear. Therefore, the observation of such a left-right asymmetric 

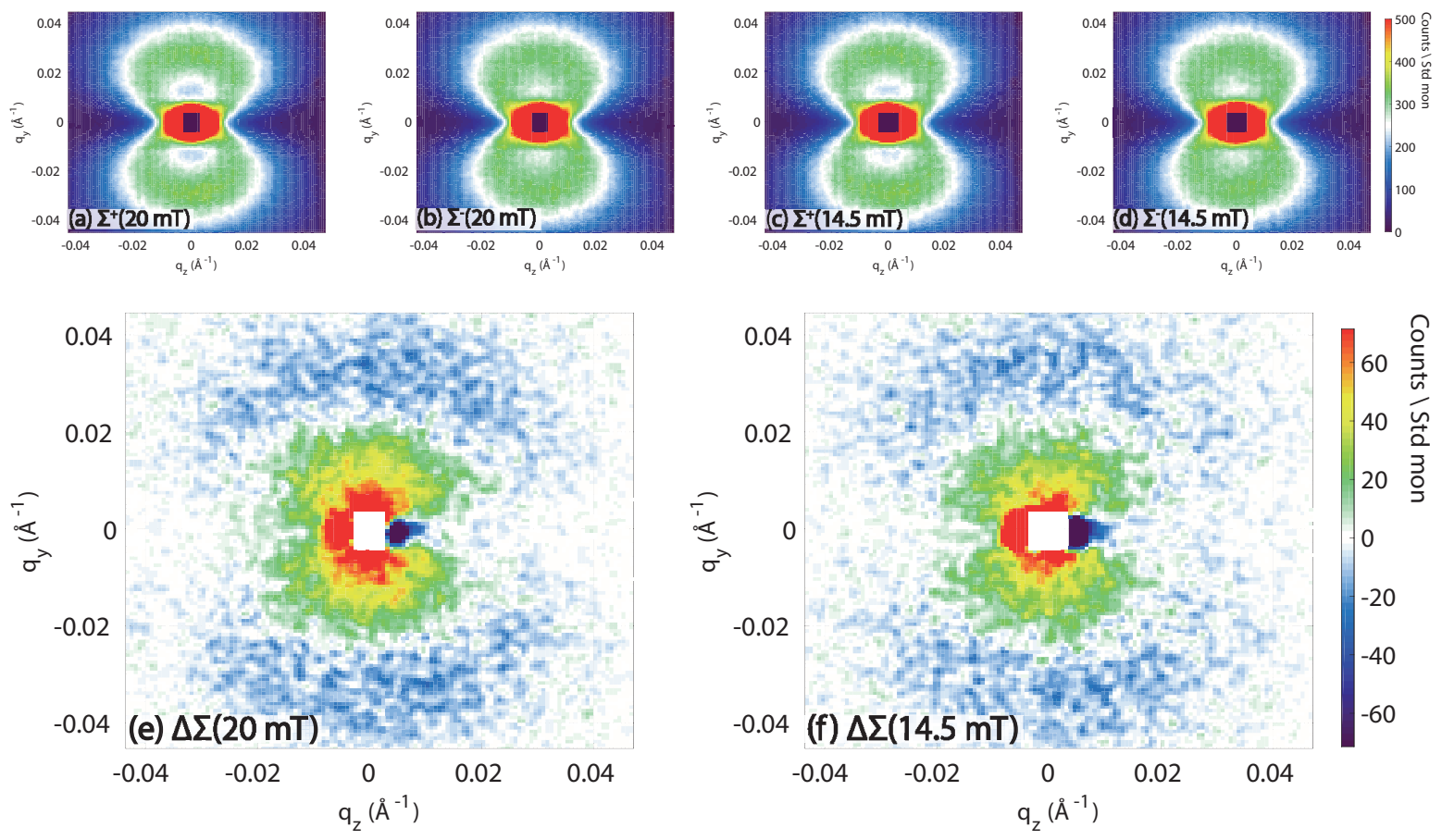

Figure 4: DMI asymmetry at $20 \mathrm{mT}$ and $14.5 \mathrm{mT}$ measured with a better statistics of 8 hours per spin channel and a smaller beam-defining aperture of $9 \times 10 \mathrm{~mm}^{2}$. (a) - (d) Flipper-off and flipper-on SANS cross sections; (e) and (f) $\Delta \Sigma=\Sigma^{+}-\Sigma^{-}$with the color scale normalized according to the aperture size to be consistent with Fig. 3(a) to (f). The difference signal amounts to $\sim 10 \%$ of the $d \Sigma^{ \pm} / d \Omega$.

SANS signal indicates that there exists an extra symmetry breaking in the system. This could possibly be related to the combined action of the macroscopic texture axis and the applied magnetic field [14].

As a final comment we would like to point out that we observe a considerable depolarization of the transmitted neutron beam by the sample, as measured with a spin analyzer [32]. This depolarization causes a contamination of a polarized SANS spin channel of one flipper state by spin leakage from the opposite flipper state. This should be quantitatively corrected and work is presently underway to resolve this issue. Qualitatively, for our case the depolarization effect results in a reduced contrast of the difference signal $I^{+}-I^{-}$, which is smaller than the real cross section difference $\Sigma^{+}-\Sigma^{-}$. The weaker the magnetic field is the stronger the expected depolarization, leading to smaller measured contrasts. Thus, the DMI asymmetry should be even more pronounced and its field dependence more significant than shown in Fig. 3(g).

\section{Conclusion}

An asymmetry has been observed in the difference of the two half polarized SANS cross sections of the nanocrystalline soft magnetic material Vitroperm. Based on a theoretical prediction for the chiral function [Eq. (5)], this observation is explained by 


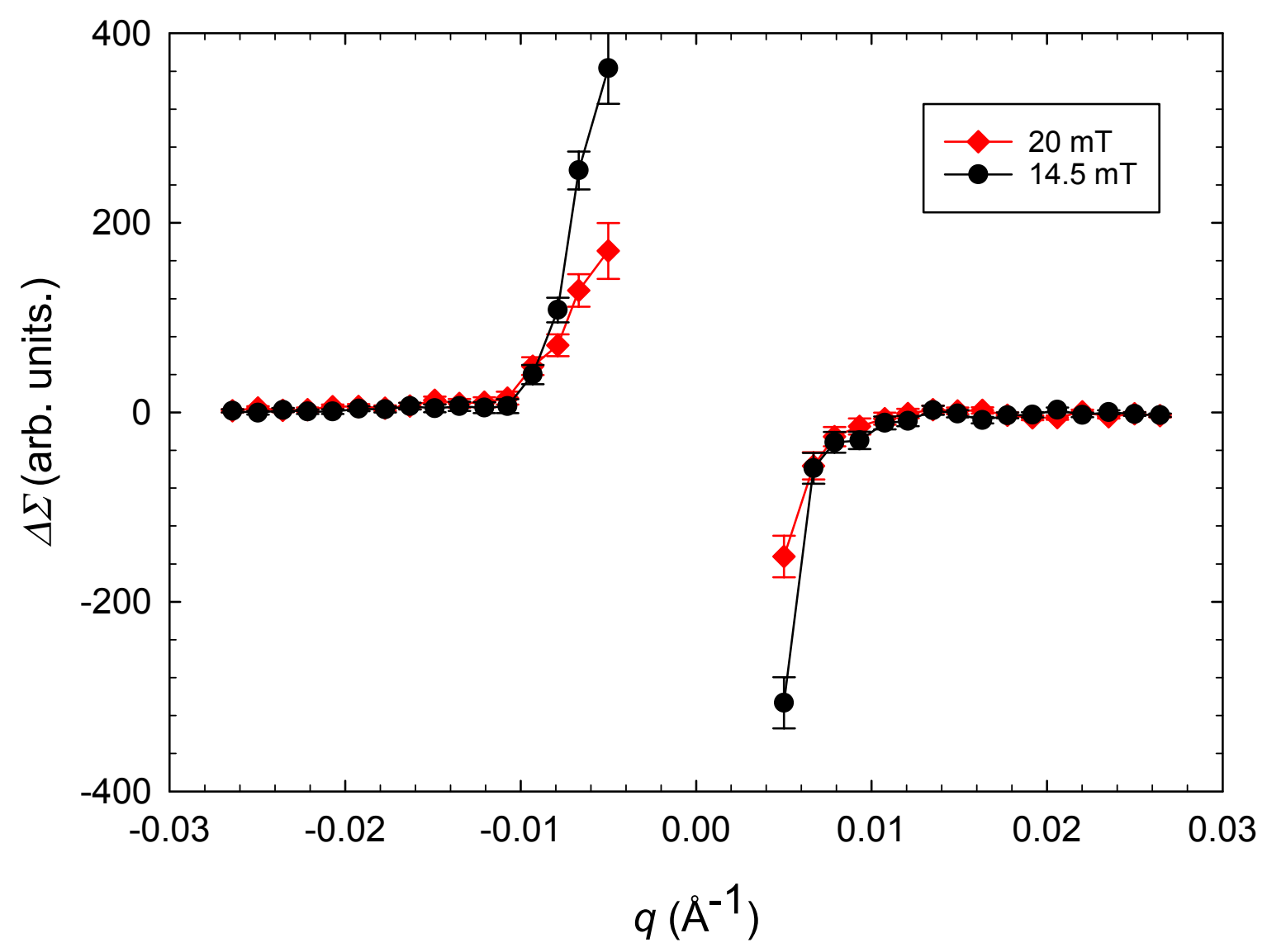

Figure 5: Azimuthally-averaged $\Delta \Sigma(q)\left( \pm 10^{\circ}\right.$ horizontal sector averages $)$ at $14.5 \mathrm{mT}$ and $20 \mathrm{mT}$.

the Dzyaloshinskii-Moriya interaction (DMI) caused by the lack of structural inversion symmetry at microstructural defect sites in the material; in our case, most likely the interface between the $\mathrm{FeSi}$ nanoparticles and the amorphous magnetic matrix. Combining this result with recent studies of nanocrystalline terbium and holmium and mechanically-deformed cobalt [21] gives strong evidence that the DMI is of general relevance for the magnetic microstructure of defect-rich ferromagnets. Equation (5) additionally predicts that in a certain magnetic field range the asymmetry will split due to the $\widetilde{M}_{z}^{2} \sin ^{2} \theta \cos \theta$ term. So far, such a contribution could not be clearly observed. Since this asymmetry would appear in a $q$-range where one expects the nuclear-magnetic interference signal, a spin analyzer would be required to perform a full polarization analysis and to eliminate the nuclear-magnetic interference term. From a theoretical point of view, it is of interest to develop appropriate expressions for defect-related DMI, rather than using the expression for cubic intrinsic DMI on which the theory [20] is based. Furthermore, the experiments demonstrate that polarized SANS is a particularly powerful tool to investigate spin chirality induced by the DMI. 


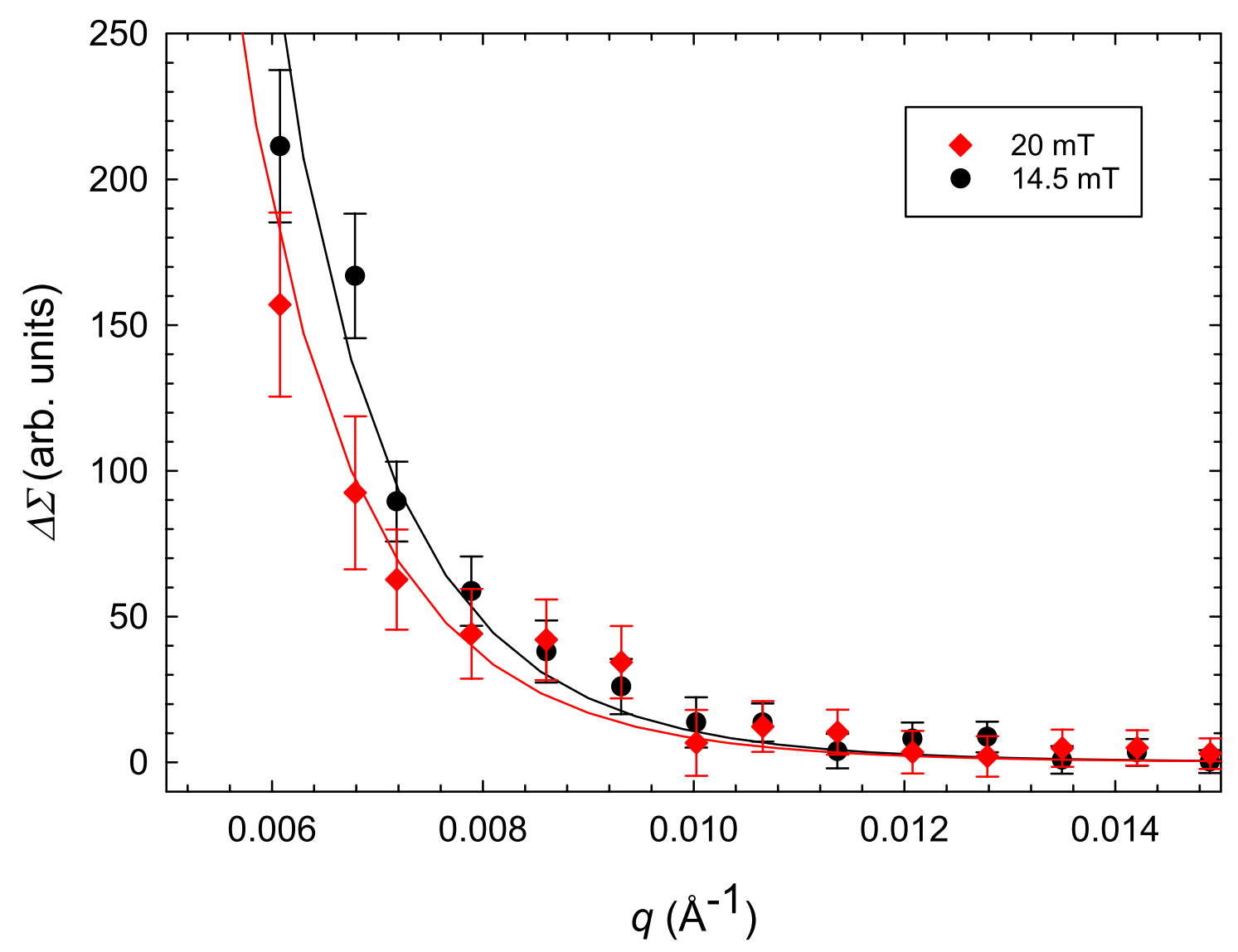

Figure 6: Estimation of the DMI strength for nanocrystalline Vitroperm. Shown is the azimuthally-averaged $\Delta \Sigma(q)$ of the $20 \mathrm{mT}$ and the $14.5 \mathrm{mT}$ data displayed in Figs. 4(e)-(f) and 5. For the analysis, the mean values of both horizontal sector averages were employed (taking into account their different signs). Solid line: Eq. (6) with no free parameters except $\left\langle H_{p}^{2}\right\rangle$ (setting $\xi_{H}=12 \mathrm{~nm}, \mu_{0} M_{s}=1.2 \mathrm{~T}, \mu_{0} H_{i}=16.5 \mathrm{mT}$ and $11.0 \mathrm{mT}, A=1.0 \times 10^{-11} \mathrm{~J} / \mathrm{m}$, and $\left.D=0.1 \mathrm{~mJ} / \mathrm{m}^{2}\right)$. The first data point next to the beam stop in Fig. 5 is excluded from the fit.

\section{Acknowledgement}

We thank Ivan Titov for carrying out the magnetization measurement. This work was supported by the Swiss National Science Foundation (Grant No. 200021/165496) and is based on experiments performed at the Swiss spallation neutron source SINQ, Paul Scherrer Institute, Villigen, Switzerland.

\section{References}

[1] I. Dzyaloshinsky. A thermodynamic theory of 'weak' ferromagnetism of antiferromagnetics. J. Phys. Chem. Solids, 4:241-255, 1958. 
[2] T. Moriya. Phys. Rev., 120:91-98, 1960.

[3] A. N. Bogdanov and D. A. Yablonskiu. Thermodynamically stable "vortices" in magnetically ordered crystals. The mixed state of magnets. Sov. Phys. JETP, 68(1):101, 1989.

[4] S. Mühlbauer, B. Binz, F. Jonietz, C. Pfleiderer, A. Rosch, A. Neubauer, R. Georgii, and P. Böni. Skyrmion lattice in a chiral magnet. Science, 323(5916):915-919, 2009.

[5] A. Fert, V. Cros, and J. Sampaio. Skyrmions on the track. Nat. Nanotech., 8(3):152-6, 2013.

[6] N. Nagaosa and Y. Tokura. Nat. Nanotech., 8:899-911, 2013.

[7] R. Wiesendanger. Nanoscale magnetic skyrmions in metallic films and multilayers: a new twist for spintronics. Nat. Mater., 1:16044, 2016.

[8] A. Chacon, L. Heinen, M. Halder, A. Bauer, W. Simeth, S. Mühlbauer, H. Berger, M. Garst, A. Rosch, and C. Pfleiderer. Nat. Phys., 14:936-941, 2018.

[9] S. Mühlbauer, D. Honecker, E. A. Périgo, F. Bergner, S. Disch, A. Heinemann, S. Erokhin, D. Berkov, C. Leighton, M. R. Eskildsen, and A. Michels. Magnetic small-angle neutron scattering. Rev. Mod. Phys., 91(1):015004, 2019.

[10] A. Arrott. Dzialoshinski-moriya interactions about defects in antiferromagnetic and ferromagnetic materials. J. Appl. Phys., 34(4):1108-1109, 1963.

[11] A. Fert and P. M. Levy. Phys. Rev. Lett., 44:1538-1541, 1980.

[12] P. M. Levy and A. Fert. J. Appl. Phys., 52:1718-1719, 1981.

[13] P. M. Levy, C. Morgan-Pond, and A. Fert. J. Appl. Phys., 53:2168-2173, 1982.

[14] V. I. Fedorov, A. G. Gukasov, V. Kozlov, S. V. Maleyev, V. P. Plakhty, and I. A. Zobkalo. Phys. Lett. A, 224:372-378, 1997.

[15] S. V. Grigoriev and Yu. O. Chetverikov and D. Lott and A. Schreyer. Phys. Rev. Lett., 100:197203, 2008.

[16] P. Beck and M. Fähnle. J. Magn. Magn. Mater., 322:3701-3703, 2010.

[17] D. A. Kitchaev, I. J. Beyerlein, and A. Van der Ven. Phys. Rev. B, 98:214414, 2018.

[18] D.-H. Kim, M. Haruta, H.-W. Ko, G. Go, H.-J. Park, T. Nishimura, D.-Y. Kim, T. Okuno, Y. Hirata, Y. Futakawa, H. Yoshikawa, W. Ham, S. Kim, H. Kurata, A. Tsukamoto, Y. Shiota, T. Moriyama, S.-B. Choe, K.-J. Lee, and T. Ono. Nat. Mater., 18:685-690, 2019.

[19] A. B. Butenko and U. K. Rößler. EPJ Web of Conferences, 40:08006, 2013.

[20] A. Michels, D. Mettus, D. Honecker, and K. L. Metlov. Effect of Dzyaloshinski-Moriya interaction on elastic small-angle neutron scattering. Phys. Rev. B, 94(5):054424, 2016.

[21] A. Michels, D. Mettus, I. Titov, A. Malyeyev, M. Bersweiler, P. Bender, I. Peral, R. Birringer, Y. Quan, P. Hautle, J. Kohlbrecher, D. Honecker, J. R. Fernández, L. F. Barquín, and K. L. Metlov. Microstructural-defect-induced Dzyaloshinskii-Moriya interaction. Phys. Rev. $B, 99(1): 014416,2019$.

[22] S. V. Maleev. Physics-Uspekhi, 45:569-596, 2002.

[23] G. Herzer. Nanocrystalline Soft Magnetic Alloys. In K. H. J. Buschow, editor, Handbook of Magnetic Materials, volume 10, pages 415-462. Elsevier, Amsterdam, 1997.

[24] G. Herzer. Acta Mater., 61:718-734, 2013.

[25] See https://vacuumschmelze.com/Search?q=vitroperm.

[26] M. Bischof, P. Staron, A. Michels, P. Granitzer, K. Rumpf, H. Leitner, C. Scheu, and H. Clemens. Acta Mater., 55:2637-2646, 2007.

[27] A. Michels, M. Elmas, F. Döbrich, M. Ames, J. Markmann, M. Sharp, H. Eckerlebe, J. Kohlbrecher, and R. Birringer. EPL (Europhysics Letters), 85:47003, 2009.

[28] Sergey Erokhin, Dmitry Berkov, and Andreas Michels. Phys. Rev. B, 92:014427, 2015.

[29] A. Michels, R. N. Viswanath, and J. Weissmüller. Domain formation and long-range spin disorder in vitroperm. Europhys. Lett., 64(1):43-49, 2003.

[30] A. Grob, S. Saranu, U. Herr, A. Michels, R. N. Viswanath, and J. Weissmüller. Phys. Status Solidi A, 201:3354-3360, 2004.

[31] V. K. Aswal, B. van den Brandt, P. Hautle, J. Kohlbrecher, J. A. Konter, A. Michels, F. M. Piegsa, J. Stahn, S. Van Petegem, and O. Zimmer. Characterisation of the polarised neutron beam at 
the small angle scattering instrument SANS-I with a polarised proton target. Nucl. Instrum. Methods Phys. Res. A, 586(1):86-89, 2008.

[32] N. Niketic, B. van den Brandt, W. Th. Wenckebach, J. Kohlbrecher, and P. Hautle. Polarization analysis in neutron small-angle scattering with a novel triplet dynamic nuclear polarization spin filter. J. Appl. Cryst., 48(5):1514-1521, 2015.

[33] M. Blume. Polarization effects in the magnetic elastic scattering of slow neutrons. Phys. Rev., 130(5):1670-1676, 1963

[34] S. V. Maleev, V. G. Bar'yakhtar, and R. A. Suris. The scattering of slow neutrons by complex magnetic structures. Sov. Phys. Solid State, 4(12):2533-2539, 1963.

[35] R. M. Moon, T. Riste, and W. C. Koehler. Polarization Analysis of Thermal-Neutron Scattering. Phys. Rev., 181(2):920-931, 1969.

[36] E. Oran Brigham. The Fast Fourier Transform. Prentice-Hall, Englewood Cliffs, 1974.

[37] Amikam Aharoni. Demagnetizing factors for rectangular ferromagnetic prisms. J. Appl. Phys., 83(6):3432-3434, 1998.

[38] André Heinemann, Helmut Hermann, Albrecht Wiedenmann, Norbert Mattern, and Klaus Wetzig. A small-angle neutron scattering model for polydisperse spherical particles with diffusion zones and application to soft magnetic metallic glass. J. Appl. Cryst., 33(6):1386-1392, Dec 2000.

[39] J. Kohlbrecher, A. Wiedenmann, and H. Wollenberger. Magnetic coupling between the different phases in nanocrystalline fe-si-b studied by small angle neutron scattering. Z. Phys. B, 104(1):14, 1997.

[40] J. Kohlbrecher. Untersuchungen an nanokristallinen weichmagnetischen Fe-Si-B Legierungen mit Kleinwinkelstreuung: Mikrostruktur, Kristallisationskinetik und magnetische Eigenschaften. PhD thesis, Technische Universität Berlin, 1996.

[41] G. Herzer. Grain structure and magnetism of nanocrystalline ferromagnets. IEEE Trans. Magn., 25(5):3327-3329, 1989.

[42] S. Seki, Y. Okamura, K. Kondou, K. Shibata, M. Kubota, R. Takagi, F. Kagawa, M. Kawasaki, G. Tatara, Y. Otani, and Y. Tokura. Magnetochiral nonreciprocity of volume spin wave propagation in chiral-lattice ferromagnets. Phys. Rev. B, 93:235131, Jun 2016. 